PROCEEDINGS OF THE

AMERICAN MATHEMATICAL SOCIETY

Volume 136, Number 7, July 2008, Pages 2473-2476

S 0002-9939(08)09140-5

Article electronically published on March 19, 2008

\title{
ON THE APPROXIMATION OF ISOLATED EIGENVALUES OF ORDINARY DIFFERENTIAL OPERATORS
}

\author{
GERALD TESCHL
}

(Communicated by Joseph A. Ball)

\begin{abstract}
We extend a result of Stolz and Weidmann on the approximation of isolated eigenvalues of singular Sturm-Liouville and Dirac operators by the eigenvalues of regular operators.
\end{abstract}

\section{INTRODUCTION}

The approximation of isolated eigenvalues of singular ordinary differential operators by the eigenvalues of regular operators is an important and well studied topic since the latter ones can be computed numerically with arbitrary precision. See the recent monograph by Zettl [8] or in particular the recent survey [7] by Weidmann.

While the case of eigenvalues below the essential spectrum is well understood, the case of eigenvalues in essential spectral gaps was only recently solved by Stolz and Weidmann in [3] (see also [4]).

Let $\tau$ be either a Sturm-Liouville expression

$$
\tau=\frac{1}{r}\left(-\frac{d}{d x} p \frac{d}{d x}+q\right),
$$

or a Dirac system

$$
\tau=\frac{1}{r}\left(\mathrm{i} \sigma_{2} \frac{d}{d x}+q\right)
$$

where $\sigma_{2}=\left(\begin{array}{cc}0 & -\mathrm{i} \\ \mathrm{i} & 0\end{array}\right)$ is the Pauli matrix, on the interval $I=(a, b)$.

As usual, we will assume the coefficients $p^{-1}, q, r$ are real-valued locally integrable functions with $p, r>0$ in the Sturm-Liouville case and $q, r$ are real, symmetric $2 \times 2$ matrices with $r>0$ in the Dirac case.

An endpoint $a$ or $b$ is called regular if it is finite and the coefficients are integrable near this endpoint. If both endpoints are regular, we will call $\tau$ regular.

Let $\mathfrak{D}(\tau)$ be the maximal domain of definition of $\tau$. Then $\tau$ is self-adjoint on $\mathfrak{D}(\tau)$ if it is limit point (l.p.) near both $a$ and $b$. Otherwise we will impose an additional boundary condition at every endpoint where $\tau$ is limit circle (l.c.). In this way we obtain a self-adjoint operator $H$ associated with $\tau$.

Received by the editors December 21, 2006, and, in revised form, February 26, 2007.

2000 Mathematics Subject Classification. Primary 34L40, 34L16; Secondary 47N50, 34B20.

Key words and phrases. Sturm-Liouville operators, Dirac operators, eigenvalues.

This research was supported by the Austrian Science Fund (FWF) under Grant No. Y330. 
A solution $\psi_{a}(z, x)\left(\psi_{b}(z, x)\right)$ of $\tau \psi=z \psi$ which is square integrable near $a(b)$ and satisfies the boundary condition at $a(b)$ (if any) is called a Weyl solution. Such a solution is unique up to a constant, and it exists at least for $z \in \mathbb{C} \backslash \sigma_{\text {ess }}(H)$.

Our aim is to approximate $H$ by regular operators $H_{n}$ obtained by restricting $\tau$ to a finite interval $\left(a_{n}, b_{n}\right) \subseteq(a, b)\left(\underline{6}\right.$. Chap. 14]). The case $a_{n}=a$ or $b_{n}=b$ is allowed.

Fix functions $u, v \in \mathfrak{D}(\tau)$. Pick $a_{n} \downarrow a, b_{n} \uparrow b$. Define $H_{n}$

$$
\begin{aligned}
H_{n}: \mathfrak{D}\left(H_{n}\right) & \rightarrow L^{2}\left(\left(a_{n}, b_{n}\right) ; r d x\right), \\
f & \mapsto \tau f
\end{aligned}
$$

where

$$
\mathfrak{D}\left(H_{n}\right)=\left\{f \in \mathfrak{D}\left(\tau_{n}\right) \mid W_{a_{n}}(u, f)=W_{b_{n}}(v, f)=0\right\} .
$$

Then Stolz and Weidmann prove the following

Theorem 1 (3). Define $H_{n}$ as above with $u=\psi_{a}\left(\lambda_{a}\right)$ and $v=\psi_{b}\left(\lambda_{b}\right)$ with $\lambda_{a}, \lambda_{b} \in\left[\lambda_{0}, \lambda_{1}\right]$ (in particular, assume that the corresponding Weyl solutions exist). Let $P_{\Omega}(H)$ be the spectral projection of $H$ corresponding to the Borel set $\Omega \subseteq \mathbb{R}$.

If $\operatorname{dim} \operatorname{Ran} P_{\left(\lambda_{0}, \lambda_{1}\right)}(H)<\infty$, then the eigenvalues of $H$ in $\left(\lambda_{0}, \lambda_{1}\right)$ are exactly the limits of eigenvalues of $H_{n}$ which lie in $\left(\lambda_{0}, \lambda_{1}\right)$. The corresponding (onedimensional) eigenprojections converge in norm.

If $\operatorname{dim} \operatorname{Ran} P_{\left(\lambda_{0}, \lambda_{1}\right)}(H)=\infty$, then the eigenvalues of $H_{n}$ accumulate in $\left(\lambda_{0}, \lambda_{1}\right)$ as $n \rightarrow \infty$.

In fact, in [3] this result is only proven in the case $\left[\lambda_{0}, \lambda_{1}\right] \cap \sigma_{\text {ess }}(H)=\emptyset$, and $\lambda_{0}, \lambda_{1}$ are not eigenvalues of $H$. Hence we will provide a proof of this slightly generalized version below.

As pointed out in [3, this result has of course one practical drawback: The Weyl solutions used to generate the boundary conditions of $H_{n}$ will not be known explicitly in general. To evade this obstacle they show that their result still holds if the Weyl solution of a nearby operator is chosen instead.

The main purpose of this note is to propose an alternate way of approximating $H$ which only involves one Weyl solution at one endpoint. More precisely, we show that if $\tau$ is l.p. at one endpoint, the Weyl solution of the other endpoint can also be chosen instead:

Theorem 2. Suppose $\tau$ is l.p. at b. Define $H_{n}$ as above with $u=\psi_{a}\left(\lambda_{a}\right)$, $\lambda_{a} \in\left[\lambda_{0}, \lambda_{1}\right]$, and $v=\psi_{a}\left(\lambda_{0}\right)$ or $v=\psi_{a}\left(\lambda_{1}\right)$ (in particular, we assume that the corresponding Weyl solutions exist).

If $\operatorname{dim} \operatorname{Ran} P_{\left(\lambda_{0}, \lambda_{1}\right)}(H)<\infty$, then the eigenvalues of $H$ in $\left(\lambda_{0}, \lambda_{1}\right)$ are exactly the limits of eigenvalues of $H_{n}$ which lie in $\left(\lambda_{0}, \lambda_{1}\right)$. The corresponding (onedimensional) eigenprojections converge in norm.

If $\operatorname{dim} \operatorname{Ran} P_{\left(\lambda_{0}, \lambda_{1}\right)}(H)=\infty$, then the eigenvalues of $H_{n}$ accumulate in $\left(\lambda_{0}, \lambda_{1}\right)$ as $n \rightarrow \infty$.

In particular, if one endpoint is regular, a solution satisfying the boundary condition at this endpoint can be taken in this case. Clearly the result cannot hold if $\tau$ is l.c. at $b$, since our assumptions contain no information on the boundary condition of $H$ at $b$ in this case.

Remark 3. (i) As shown in [3], if $\tau$ is l.c. at $a$, then $u$ can be choosen to be any function in $\mathfrak{D}(\tau)$ generating the boundary condition of $H$ at $a$. 
(ii) The same result (with the same proof) holds for Jacobi operators (see [5]).

\section{Approximation by Regular operators}

We begin by recalling that $H_{n}$ converges to $H$ strongly ([6]). Strictly speaking this statement makes no sense since $H_{n}$ and $H$ live in different Hilbert spaces. This can be easily fixed by using $\alpha \mathbb{1} \oplus H_{n} \oplus \alpha \mathbb{1}$ on $L^{2}((a, b) ; r d x)=L^{2}\left(\left(a, b_{n}\right) ; r d x\right) \oplus$ $L^{2}\left(\left(a_{n}, b_{n}\right) ; r d x\right) \oplus L^{2}\left(\left(a_{n}, b\right) ; r d x\right)$ where $\alpha$ is a fixed real constant outside $\left[\lambda_{0}, \lambda_{1}\right]$. Alternatively, one can also use generalized strong convergence as introduced in $[3]$.

Lemma 4. Suppose that either $H$ is limit point at a or that $u=\psi_{-}\left(\lambda_{0}\right)$ for some $\lambda_{0}$ and, similarly, that either $H$ is limit point at $b$ or $v=\psi_{+}\left(\lambda_{1}\right)$ for some $\lambda_{1}$. Then $H_{m}$ converges to $H$ in strong resolvent sense as $m \rightarrow \infty$.

In addition, we need the following abstract result. In this respect we remark that for a self-adjoint projector $P$ we have

$$
\operatorname{dim} \operatorname{Ran}(P)=\operatorname{tr}(P)=\|P\|_{1},
$$

where $\|\cdot\|_{1}$ denotes the trace class norm. If $P$ is not finite rank, then it is of course not trace class, and all three numbers are equal $\infty$.

Lemma 5. Let $A_{n}, A$ be self-adjoint operators such that $A_{n} \rightarrow A$ in strong resolvent sense. Then

$$
\operatorname{tr}\left(P_{\left(\lambda_{0}, \lambda_{1}\right)}(A)\right) \leq \liminf \operatorname{tr}\left(P_{\left(\lambda_{0}, \lambda_{1}\right)}\left(A_{n}\right)\right)
$$

If in addition, $\operatorname{tr}\left(P_{\left(\lambda_{0}, \lambda_{1}\right)}\left(A_{n}\right)\right) \leq \operatorname{tr}\left(P_{\left(\lambda_{0}, \lambda_{1}\right)}(A)\right)$, then

$$
\lim _{n \rightarrow \infty} \operatorname{tr}\left(P_{\left(\lambda_{0}, \lambda_{1}\right)}\left(A_{n}\right)\right)=\operatorname{tr}\left(P_{\left(\lambda_{0}, \lambda_{1}\right)}(A)\right)
$$

and if $\operatorname{tr}\left(P_{\left(\lambda_{0}, \lambda_{1}\right)}(A)\right)<\infty$ we even have

$$
\lim _{n \rightarrow \infty}\left\|P_{\left(\lambda_{0}, \lambda_{1}\right)}\left(A_{n}\right)-P_{\left(\lambda_{0}, \lambda_{1}\right)}(A)\right\|_{1}=0 .
$$

Proof. The first part is just Lemma 5.2 from [1]. This also implies the second if $\operatorname{tr}\left(P_{\left(\lambda_{0}, \lambda_{1}\right)}(A)\right)=\infty$. Otherwise, if $\operatorname{tr}\left(P_{\left(\lambda_{0}, \lambda_{1}\right)}(A)\right)<\infty$, we have

$$
\lim \sup \operatorname{tr}\left(P_{\left(\lambda_{0}, \lambda_{1}\right)}\left(A_{n}\right)\right) \leq \operatorname{tr}\left(P_{\left(\lambda_{0}, \lambda_{1}\right)}(A)\right)
$$

and the first claim follows. The second is then a consequence of Grümm's theorem ([2, Thm. 2.19]).

Now it remains to show that this result is applicable in our situation.

Lemma 6. Let $H_{n}$ be defined as in (3) with

(i) $u=\psi_{a}\left(\lambda_{a}\right), v=\psi_{b}\left(\lambda_{b}\right)$ with $\lambda_{a}, \lambda_{b} \in\left[\lambda_{0}, \lambda_{1}\right]$ or

(ii) $u=\psi_{a}\left(\lambda_{a}\right)$ with $\lambda_{a} \in\left[\lambda_{0}, \lambda_{1}\right]$ and $v=\psi_{a}\left(\lambda_{b}\right)$ with $\lambda_{b} \in\left\{\lambda_{0}, \lambda_{1}\right\}$. Then,

$$
\operatorname{tr}\left(P_{\left(\lambda_{0}, \lambda_{1}\right)}\left(H_{n}\right)\right) \leq \operatorname{tr}\left(P_{\left(\lambda_{0}, \lambda_{1}\right)}(H)\right) .
$$

Proof. Abbreviate $P=P_{\left(\lambda_{0}, \lambda_{1}\right)}(H), P_{n}=P_{\left(\lambda_{0}, \lambda_{1}\right)}\left(H_{n}\right)$.

(i) Since this part is identical to the proof in [3], we just give an outline. Let $\tilde{\psi}_{1}, \ldots, \tilde{\psi}_{k} \in \operatorname{Ran} P_{n}$ be the normalized eigenfunctions of $H_{n}$, and construct

$$
\psi_{j}(x)=\left\{\begin{array}{cl}
\gamma_{a, j} u(x), & x<a_{n}, \\
\tilde{\psi}_{j}(x), & a_{n} \leq x \leq b_{n}, \\
\gamma_{b, j} v(x), & x>b_{n},
\end{array}\right.
$$


where $\gamma_{a, j}, \gamma_{b, j}$ are chosen such that $\psi_{j} \in \mathfrak{D}(\tau)$. A computation now shows that

$$
\left\|\left(H-\frac{\lambda_{1}+\lambda_{0}}{2}\right) \psi\right\|<\frac{\lambda_{1}-\lambda_{0}}{2}\|\psi\|
$$

for any $\psi$ in the linear span of the $\psi_{j}$ 's, which yields the first result.

(ii) By considering two steps from $\left(a_{n}, b_{n}\right)$ to $\left(a, b_{n}\right)$ and from $\left(a, b_{n}\right)$ to $(a, b)$, we see that the first step is covered by (i), and hence it is no restriction to assume $a_{n}=a$. Now proceed as in the previous case but use

$$
\psi_{j}(x)=\left\{\begin{array}{cc}
\tilde{\psi}_{j}(x)-\gamma_{j} v(x), & x \leq b_{n}, \\
0, & x>b_{n}
\end{array}\right.
$$

where $\gamma_{j}$ are chosen such that $\psi_{j} \in \mathfrak{D}(\tau)$. Now let $\psi=\sum_{j} c_{j} \psi_{j}$ be in the linear span of the $\psi_{j}$ 's. Then, since $v$ is also an eigenvector of $H_{n}$ and hence orthogonal to the $\psi_{j}$ 's, we have

$$
\begin{aligned}
\left\|\left(H-\frac{\lambda_{1}+\lambda_{0}}{2}\right) \psi\right\|^{2} & =\left\|\sum_{j} c_{j}\left(\frac{2 \lambda_{j}-\lambda_{1}-\lambda_{0}}{2} \tilde{\psi}_{j}(x)-\frac{2 \lambda_{b}-\lambda_{1}-\lambda_{0}}{2} \gamma_{j} v(x)\right)\right\|^{2} \\
& =\sum_{j}\left|c_{j}\right|^{2}\left(\frac{2 \lambda_{j}-\lambda_{1}-\lambda_{0}}{2}\right)^{2}+|\gamma|^{2}\left(\frac{\lambda_{1}-\lambda_{0}}{2}\right)^{2}\|v\|_{\left(a, b_{n}\right)}^{2} \\
& <\left(\frac{\lambda_{1}-\lambda_{0}}{2}\right)^{2}\|\psi\|^{2},
\end{aligned}
$$

where $\gamma=\sum_{j} c_{j} \gamma_{j}$. Hence the second result follows.

Theorem 1 and Theorem 2 now follow by combining the last two lemmas.

\section{ACKNOWLEDGMENTS}

I thank Helge Krüger for discussions.

\section{REFERENCES}

[1] F. Gesztesy, B. Simon, and G. Teschl, Zeros of the Wronskian and renormalized oscillation theory, Amer. J. Math. 118, 571-594 (1996). MR.1393260 (97g:34105)

[2] B. Simon, Trace Ideals and Their Applications, 2nd ed., Amer. Math. Soc., Providence, RI, 2005. MR2154153 (2006f:47086)

[3] G. Stolz and J. Weidmann, Approximation of isolated eigenvalues of ordinary differential operators, J. Reine und Angew. Math. 445, 31-44 (1993). MR.1244968 (95a:34122)

[4] G. Stolz and J. Weidmann, Approximation of isolated eigenvalues of general singular ordinary differential operators, Results Math. 28, no. 3-4, 345-358 (1995). MR.1356897(96j:34145)

[5] G. Teschl, Jacobi Operators and Completely Integrable Nonlinear Lattices, Math. Surv. and Mon. 72, Amer. Math. Soc., Providence, RI, 2000. MR1711536 (2001b:39019)

[6] J. Weidmann, Spectral Theory of Ordinary Differential Operators, Lecture Notes in Mathematics 1258, Springer-Verlag, Berlin, 1987. MR923320 (89b:47070)

[7] J. Weidmann, Spectral theory of Sturm-Liouville operators; approximation by regular problems, in Sturm-Liouville Theory: Past and Present (eds. W. Amrein, A. Hinz and D. Pearson), 75-98, Birkhäuser, Basel, 2005. MR2145078

[8] A. Zettl, Sturm-Liouville Theory, Math. Surv. and Mon. 121, Amer. Math. Soc., Providence, RI, 2005. MR2170950 (2007a:34005)

Faculty of Mathematics, Nordbergstrasse 15, 1090 Wien, Austria; and International Erwin Schrödinger Institute for Mathematical Physics, Boltzmanngasse 9, 1090 Wien, Austria

E-mail address: Gerald.Teschl@univie.ac.at 